\title{
Comparación del tratamiento con placas en fractura de tibia distal
}

\author{
Comparison of treatment with plates for distal tibia fractures
}

\author{
Marroquín-Herrera O,* García-Balderas A, ${ }^{\ddagger}$ Ortega-Meza E, ${ }^{\S}$ \\ Aburto-González P, "Rodríguez-Albístegui C," Olvera-Vásquez R"
}

Hospital General de Querétaro.

RESUMEN. Introducción: Actualmente el tratamiento quirúrgico de fracturas de tibia distal extraarticular es controversial por las múltiples técnicas y materiales de osteosíntesis, las técnicas Mínimo Invasivo Percutánea (MIPO) nos permiten preservar la circulación perióstica, punto crucial en los procesos naturales de convalecencia del paciente, por ello esta técnica es de gran relevancia en nuestro estudio. Objetivo: Observar que este abordaje de fracturas de tibia distal tiene menos complicaciones y mejor resultado funcional que la fijación con técnica abierta. Material y métodos: Se realizó un estudio de tipo longitudinal, ambispectivo y analítico en el período de Julio de 2018 a Septiembre de 2019 en pacientes adultos con fractura cerrada de tibia distal extraarticular sin comorbilidades, el cálculo se realizó con el programa WinPEPI (Programs for Epidemiologists for Windows) versión 11.43 basado en el estudio de Paul Toogooda, con un intervalo de confianza de $95 \%$ se obtuvo un total mínimo de 20 pacientes por cada grupo. Se usaron medidas de tendencia central, correlaciones entre técnica y variable. Resultados: Se analizaron dos grupos de 20 pacientes comparando la técnica MIPO vs. la convencional en un período de seis meses postquirúrgico evaluando riesgo de infección, dehiscencia de herida, consolidación ósea radiográfica, funcionalidad articular y datos demográficos, mostrando superioridad estadística positiva para la técnica MIPO. Conclusiones: Se obtuvieron datos relevantes a favor de
ABSTRACT. Introduction: Currently the surgical treatment of fractures of extraarticular distal tibia is controversial for the multiple techniques and materials of osteosynthesis, the Minimally Invasive Percutaneous Techniques (MIPO) allow us to preserve the periosteal circulation, crucial point in the natural processes of convalescence of the patient, so this technique takes relevance in our study. Objective: To observe that this approach to fractures of the distal tibia has fewer complications and better functional result than fixation with open technique. Material and methods: A longitudinal, ambispective and analytical study was conducted in the period from July 2018 to September 2019 in patients with closed fracture of the extraarticular distal tibia in patients over 18 years of age without previously diagnosed musculoskeletal comorbidities, the calculation was performed with the WinPEPI program (Programs for Epidemiologists for Windows) version 11.43 based on the study by Paul Toogooda et al (2018) with a confidence interval of $95 \%$ a minimum total of 20 patients for each group. Measures of central tendency, correlations between technique and variable were used. Results: Two groups of 20 patients were analyzed comparing MIPO vs conventional technique in a period of 6 months postsurgical evaluating risk of infection, wound dehiscence, radiographic bone consolidation, joint functionality and demographic data; showing positive statistical superiority for MIPO technique.

\section{Nivel de evidencia: IV}

* Médico Especialista en Traumatología y Ortopedia.

‡ Maestro en Ciencias; Médico Especialista en Traumatología y Ortopedia.

$\S$ Médico Especialista en Traumatología y Ortopedia, Cirujano de Columna Vertebral.

" Médico Especialista en Traumatología y Ortopedia.

Correspondencia:

Omar Marroquín Herrera

Hospital General de Querétaro, Av. 5 de Febrero 101, Colonia Los Virreyes, 75859.

E-mail: granleag@hotmail.com

Recibido: 15-10-2020. Aceptado: 15-01-2021.

Citar como: Marroquín-Herrera O, García-Balderas A, Ortega-Meza E, Aburto-González P, Rodríguez-Albístegui C, Olvera-Vásquez R. Comparación del tratamiento con placas en fractura de tibia distal. Acta Ortop Mex. 2021; 35(1): 40-45. https://dx.doi.org/10.35366/100929 
MIPO con consolidación ósea y mayor puntaje funcional articular a los seis meses.

Palabras clave: Tibia, técnica, mínimo invasivo percutáneo, convencional, circulación perióstica.

\section{Introducción}

Hablar de fracturas de tibia distal obliga a conocer los principales reparos anatómicos, dentro de ellos se considera a la tibia distal, junto con el peroné y astrágalo, la articulación del tobillo, que participa en la formación de la articulación sindesmal. Toman relevancia las estructuras anatómicas en el compartimento anterior y lateral de la pierna distal. Müller definió en 1990 la metáfisis de tibial distal construyendo un cuadrado con los lados de longitud definidos por la porción más ancha del plafón tibial; las fracturas extraarticulares de la tibia distal corresponden a tipo 43A de acuerdo con la Clasificación de la Asociación de Trauma Ortopédico (AO/OTA 1996). ${ }^{{ }^{2}}$

Las fracturas de tibia distal comprenden $0.7 \%$ de todas las fracturas con una incidencia anual de 7.9 por cada 10,000 adultos. En adultos jóvenes, la causa más común es traumatismo de alta energía como un accidente automovilístico o una lesión deportiva. Las fracturas en adultos mayores a menudo resultan de traumas de baja energía como caídas de su propia altura que reflejan osteopenia subyacente y osteoporosis. . $2,3,4^{2}$

La necesidad de una fijación biológica condujo al desarrollo del concepto de fijador interno bloqueado, consiste en que la cabeza del tornillo se engancha en el orificio de la placa, de esta manera la transferencia de carga del hueso a la placa se realiza mediante el mecanismo de bloqueo del tornillo. El desarrollo de placas bloqueadas logra una estabilidad mecánica angular y axial en la interfaz entre la cabeza del tornillo de bloqueo y el orificio de bloqueo en la placa, creando un dispositivo de ángulo fijo, el principio de fijación es el de una fijación externa con una barra de fijación (placa) minimizada a la distancia ósea, este principio de fijación tiene implicaciones importantes para la estabilidad mecánica y la amplia aplicación clínica de construcciones de placas bloqueadas, donde la compresión de la placa en el hueso ya no es necesaria y la placa se puede mantener elevada a una pequeña distancia del hueso, evitando así el deterioro de la perfusión sanguínea y prevención del aplastamiento perióstico. La estabilidad no depende del ajuste apretado de la placa en la superficie del hueso y las placas de bloqueo no necesitan ser contorneadas individualmente, sino que se pueden usar en su forma precontorneada fabricada, todos los tornillos bloqueados actúan juntos y distribuyen la carga de manera más uniforme a lo largo de la placa en comparación con los tornillos en el revestimiento convencional, para contrarrestar las fuerzas
Conclusion: Relevant data were obtained in favor of MIPO with bone consolidation and higher functional score at six months.

Keywords: Tibia, technique, minimally invasive percutaneous, conventional, perióstica circulation.

axiales los tornillos de bloqueo experimentan un momento de flexión como una construcción de viga única. ${ }^{5}$ Por lo tanto, necesitan tener un diámetro mayor para soportar las cargas de flexión, esto permite un enfoque mínimamente invasivo para la reducción de fractura de manera indirecta y la aplicación de placas de bloqueo. Las placas pueden insertarse a través de una incisión pequeña en la piel y luego deslizarse a lo largo de la superficie del hueso. La técnica evolucionó hasta la generación actual de placas de bloqueo de diseño anatómico y con estas características apuntan a la estabilidad rígida para permitir movilización postoperatoria temprana y pronto retorno a la función, así como la rigidez adecuada para estimular la curación de fracturas de eje multifragmentario complejo y fracturas articulares en diferentes ubicaciones anatómicas.

A este respecto, el mecanismo de bloqueo poliaxial ofrece la opción de anclaje suficiente, incluso para patrones de fractura conminuta con múltiples fragmentos de hueso. Con la aplicación mínimamente invasiva de placas de bloqueo anatómicas, el suministro de sangre al periostio y al área de la fractura se conserva en gran medida, por lo que constituye un medio biológico para la curación de la fractura, esto favorece una curación ósea adecuada y menor riesgo de infecciones, unión tardía o pérdida secundaria de reducción. La diferencia más importante entre las placas convencionales y las placas bloqueadas es el entorno mecánico que generan ambos principios de fijación, para que la osteosíntesis convencional resulte exitosa, los puentes osteonales y las brechas de fractura deben ser menores de $0.5 \mathrm{~mm}$ y la tensión interfragmentaria resultante debe permanecer por debajo de $2 \%$. Las placas bloqueadas están indicadas para el tratamiento de fracturas en situaciones en que los fragmentos no están en contacto directo entre sí, aun con huecos más grandes la curación puede durar más, incluso los huecos de fractura mayores de $2 \mathrm{~mm}$ todavía son capaces de consolidar; en contraste con la estabilidad absoluta en la osteosíntesis convencional, la osteosíntesis bloqueada requiere una estabilidad relativa con movimiento interfragmentario en el espacio de fractura que puede superar fácilmente $2 \%$ de la tensión interfragmentaria. ${ }^{6}$

La técnica MIPO para las fracturas de tibia distal es más adecuada para pacientes que presentan algún tipo de lesión dérmica o riesgo de comorbilidades dérmicas, donde la disección extensa conllevaría un alto riesgo de complicaciones de la herida. La técnica MIPO está más reservada para las fracturas de la tibia distal en las que el plafón no presenta conminución severa. 
Todos los métodos de fijación tienen méritos y deméritos, por lo tanto no hay consenso para la superioridad de un método sobre el otro, la curación de tejidos blandos y consolidación ósea es de suma importancia, las técnicas de deslizamiento mínimamente invasivas reducen el riesgo de lesiones de tejido blando y mantienen un ambiente más favorable biológicamente para la curación de fracturas, los resultados dependen de la gravedad de la lesión de tejidos blandos, sincronización quirúrgica, técnica quirúrgica y comorbilidades del paciente. Además la literatura apoya que un puente de piel de $7 \mathrm{~cm}$ debe estar presente entre una y otra incisión en caso de realizarse osteosíntesis de peroné, en el cual se prefiere un bordaje posterolateral. ${ }^{8}$

\section{Material y métodos}

Estudio longitudinal, ambispectivo y analítico en el período de Julio de 2018 a Septiembre de 2019 en pacientes con fractura de tibia distal extraarticular que fueron intervenidos con osteosíntesis con placa anterolateral. El cálculo de la muestra se realizó con en el programa WinPEPI (Programs for Epidemiologists for Windows) versión 11.43 basado en el estudio de Paul Toogood y colaboradores. Con un intervalo de confianza de $95 \%$ se obtuvo un total mínimo de 20 pacientes por cada grupo.

Criterios de inclusión: pacientes mayores de 18 años de edad que aceptaron y firmaron el consentimiento informado de procedimiento quirúrgico.

Criterios de exclusión: fracturas expuestas de tibia distal, con enfermedades siquiátricas, pacientes con enfermedades neuromusculares degenerativas y pacientes con diabetes mellitus diagnosticados previamente.

Este estudio siguió las normas éticas que dicta la declaración de Helsinki en la 64 Asamblea General, Fortaleza, Brasil, Octubre 2013 así como a lo dictaminado por la Ley General de Salud y al Reglamento de la ley General de Salud en materia de experimentación en seres humanos y a las Normas Oficiales Mexicanas. La técnica quirúrgica propuesta en este protocolo está científicamente aceptada y se utiliza a nivel internacional. Los resultados obtenidos en el presente estudio son estrictamente confidenciales y su uso es exclusivo para fines académicos.

Métodos estadísticos: se utilizó estadística descriptiva con promedios, desviación estándar, frecuencias absolutas y relativas, correlacionando con la prueba de $\chi^{2}$ y la correlación de Spearman, con un nivel de confianza de $95 \%$.

Se revisó el expediente e historia clínica del mecanismo de fractura de todos los pacientes para determinar si cumplían con los criterios de inclusión. Posteriormente se explicó con detalle el procedimiento quirúrgico propuesto, dándoles a firmar el consentimiento informado.

Se realizó incisión a nivel de tibia distal de lateral a medial de $4 \mathrm{~cm}$ longitud, exponiendo tejido celular subcutáneo, disección roma, se incide con técnica de bandera retináculo extensor proximal, se expone tendón tibial anterior, tendón extensor de primer ortejo y parcialmente extensor común de los dedos, se identifica nervio peroneo profundo y arteria terminal tibial anterior, los cuales se refieren a lateral con separador de Hoffman, se identifica plano entre tibia y músculo tibial anterior, lugar donde se procede a deslizar placa LCP anatómica de tibia distal anterolateral bajo control fluoroscópico; se realiza fijación con tornillo cortical $3.5 \mathrm{~mm}$ anteroposterior bicortical para lograr coaptación de placa a tibia distal; bajo fluroscopio reducción de trazo de fractura con adecuada alineación, longitud y rotación, procediendo a efectuar incisión lateral proximal en pierna a nivel de tres orificios proximales de placa, lugar donde se coloca tornillo bicortical $3.5 \mathrm{~mm}$ corroborando adecuada coaptación de interface placa hueso por fluoroscopio; continuamos con colocación de pernos de bloqueo $3.5 \mathrm{~mm}$ a nivel proximal y distal para mantener principios de estabilidad relativa de osteosíntesis y principio biomecánico de sostén, se finaliza procedimiento quirúrgico con reparación de retináculo extensor con Vicryl 2.0, afrontamiento de tejido subcutáneo con Vicryl 2.0, se finaliza con puntos simples de Nylon 2-0 así como colocación de vendaje simple.

\section{Resultados}

Se estudiaron 40 pacientes, los cuales se dividieron en dos grupos de estudio con base en el abordaje y técnica quirúrgica de osteosíntesis con placa anterolateral distal; grupo MIPO y grupo convencional.

En el grupo MIPO el promedio de edad fue de 41.65 años (rango de 24 a 67 años) presentando una desviación estándar de 10.96; de manera similar en el grupo convencional la edad promedio fue de 38.25 años (rango de 19 a 68 años) presentando una desviación estándar de 13.23 años.

La distribución de géneros fue para el grupo MIPO de 15 hombres $(75 \%)$, cinco mujeres $(25 \%)$ y para el grupo convencional 12 hombres $(60 \%)$, ocho mujeres $(40 \%)$.

Se calculó la tasa de incidencia acumulada para infección de herida quirúrgica en grupo MIPO a las 24 horas (0), dos semanas (0.05), seis semanas (0) y el grupo convencional a las 24 horas (0.05), dos semanas $(0.20)$, seis semanas (0.05); obteniendo un riesgo relativo total a las dos semanas de 0.25 . Al aplicar la prueba de $\chi^{2}$ se encontró que la incidencia de infección no mostró dependencia estadísticamen-

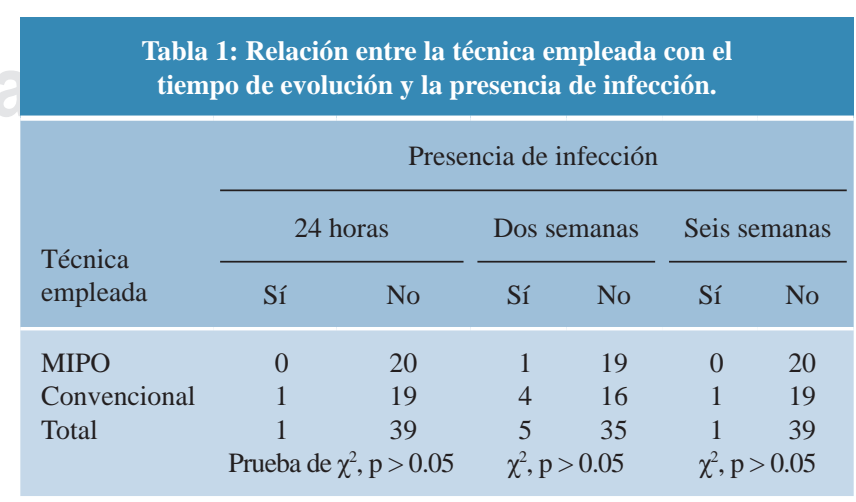


te significativa $(p>0.05)$ con las técnicas quirúrgicas en las primeras seis semanas postquirúrgicas (Tabla 1).

Se calculó la tasa de incidencia acumulada para dehiscencia de herida quirúrgica en el grupo MIPO a las 24 horas (0), dos semanas (0), seis semanas (0) y en el grupo convencional a las 24 horas (0.05), dos semanas (0.25), seis semanas (0.15); obteniendo relevancia con un riesgo relativo a las dos semanas (0.25). La aplicación de la prueba de $\chi^{2}$ mostró que a las dos semanas hay dependencia entre la técnica quirúrgica y la incidencia dehiscencia de herida quirúrgica a favor del grupo MIPO estadísticamente significativo $(\mathrm{p}<0.05)$.

La tasa de incidencia acumulada para consolidación ósea en el grupo MIPO a las seis semanas (0.85), tres meses (0.70), seis meses (1.00) y en el grupo convencional a las seis semanas $(0.40)$, tres meses $(0.45)$, seis meses $(0.55)$. $\mathrm{Al}$ aplicar el coeficiente de contingencia observamos una correlación moderada positiva y significativa, a las seis semanas ( $C C=0.421, \mathrm{p}<0.05)$, tres meses $(\mathrm{CC}=0.313$, $\mathrm{p}<$ $0.05)$, seis meses $(C C=0.474, p<0.05)$, en la influencia de la técnica de osteosíntesis para favorecer una consolidación ósea en menor tiempo con la técnica MIPO.

La tasa de incidencia acumulada para funcionalidad articular en el grupo MIPO a las seis semanas (0.9), tres meses (1), seis meses (1) y para el grupo convencional a las seis semanas (0.2), tres meses (0.65), seis meses (0.8). La correlación observada para la técnica quirúrgica tiene una gran repercusión en el grado de funcionalidad articular a la semana seis $(C C=0.0421, \mathrm{p}<0.05)$, tres meses $(\mathrm{CC}=0.313$, $\mathrm{p}$ $<0.05$ ), seis meses $(\mathrm{CC}=0.474, \mathrm{p}<0.05)$ todo esto a favor de la técnica MIPO (Tabla 2).

\section{Discusión}

La colocación de placa anterolateral con la técnica MIPO versus abordaje convencional ha demostrado resultados a favor de la técnica MIPO, esto justifica la menor lesión de tejidos, preservación de circulación así como el principio de estabilidad relativa, la cual genera consolidación secundaria; se discute el uso de tornillo interfragmentario a través de la placa, se han observado resultados con mayor éxito en cuanto a la disminución del riesgo de no unión o retardo en la consolidación; un estudio apoya el uso de un tornillo interfragmentario en combinación con la placa, esto se refiere a tornillo a través de la placa, el cual conduce a una curación radiológica significativamente más rápida con menos complicaciones; añadir más de un tornillo de fijación no parece aportar ningún beneficio adicional con respecto al tiempo de consolidación radiográfica o el tiempo para soportar todo el peso, pero puede ayudar en la reducción y resistencia a la carga máxima. Finalmente, agregar un tornillo interfragmentario no necesariamente asegura absoluta estabilidad, pero tiene sus ventajas como ayuda técnica en la reducción. 9,10,11,12

Un estudio prospectivo doble ciego realizado en China en dos grupos de 50 pacientes cada uno comparan técnica
MIPO vs. convencional abierta incluyendo fracturas de tibia distal de tipo A, B y C, el cual arroja resultados similares en cuanto a tiempo de consolidación para tipo A y B; sin embargo, los resultados para lesiones tipo $\mathrm{C}$ fueron mejores, disminuyen el tiempo de consolidación, las complicaciones en tejidos blandos así como la pronta incorporación a las actividades de la vida diaria. ${ }^{13}$ Otro estudio retrospectivo de 70 pacientes con manejo abierto arrojó resultados negativos, presentando una tasa de complicaciones de tejidos blandos y consolidación en $60 \%$ así como una escala AOFAS de 73 a los dos años de seguimiento, por lo cual sugieren buscar nuevas alternativas de tratamiento quirúrgico. ${ }^{14,15}$

La realización de la técnica MIPO para osteosíntesis en extremidad superior e inferior es un procedimiento nuevo, el cual cambia algunos conceptos de osteosíntesis, por ejemplo, la reducción anatómica con compresión interfragmentaria para generar estabilidad absoluta de trazos metadiafisarios y de esta forma generar una consolidación primaria. ${ }^{11,17}$ Los nuevos avances en el estudio de procesos de consolidación secundaria por estabilidad relativa y la preservación de la irrigación perióstica generada al realizar técnicas deslizadas nos lleva a inferir la gran utilidad de estos nuevos procedimientos. ${ }^{16,17}$

$\mathrm{Al}$ contrario de lo mencionado anteriormente, se encontró un metaanálisis que compara el manejo de fracturas de tibia distal con placas anterolateral en la variante de la técnica MIPO vs. la convencional, el cual arroja resultados similares en tiempo de consolidación así como escala funcional para tobillo y muestra una superioridad mínima en cuanto a lesión de tejidos blandos a favor de la técnica MIPO; del mismo modo detalla mayor tiempo quirúrgico así como mayor uso de fluroscopía, situación desfavorable de la técnica MIPO. ${ }^{18}$

Históricamente, las complicaciones de la herida han sido documentadas hasta en $36 \%$ después de la fijación interna con reducción abierta (ORIF) en fracturas del pilón tibial, con infecciones reportadas de 37 a 55\%; la morbilidad de la rodilla de tipo neuralgia se ha reportado hasta en $71 \%$ en la colocación de clavo centromedular anterógrado así como la mala alineación del eje tibial, trayendo como consecuencia el cambio en los puntos de apoyo y fases de la marcha. ${ }^{7}$ Toma especial relevancia el reporte de varios artículos sobre la técnica MIPO de placas mediales, las cuales presentan una alta incidencia de lesión dérmica, riesgo de exposición de material de osteosíntesis a corto y mediano plazo con la necesidad de retiro del material de osteosíntesis, por ello se pone en marcha el uso de nuevos abordajes de la fractura que contengan mejor cobertura de material de osteosíntesis llevando a un abordaje lateral de tipo MIPO, el cual proporciona una apropiada reducción y estabilidad de la fractura, mantiene un ambiente biológicamente amigable para la curación de fracturas y disminuye las complicaciones de la herida a través de la creación de incisiones más pequeñas tomando en cuenta la rigidez de la placa en carga tanto axial como torsional probada con modelos de hueso sintético, que fueron creados para 
Tabla 2: Grado de consolidación ósea radiográfica en pacientes con técnica MIPO vs. convencional, 2019. (N = 40).

\begin{tabular}{|c|c|c|c|c|c|c|c|}
\hline \multirow[b]{2}{*}{ Factor } & \multicolumn{2}{|c|}{ Seis semanas } & \multicolumn{3}{|c|}{ Tres meses } & \multicolumn{2}{|c|}{ Seis meses } \\
\hline & Sin consolidación & $\begin{array}{c}\text { Velado } \\
\text { radiográfico }\end{array}$ & $\begin{array}{c}\text { Velado } \\
\text { radiográfico }\end{array}$ & Puentes óseos & $\begin{array}{l}\text { Consolidación } \\
\text { completa }\end{array}$ & Puentes óseos & $\begin{array}{c}\text { Consolidación } \\
\text { completa }\end{array}$ \\
\hline MIPO & 3 & 17 & 5 & 14 & 1 & 0 & 20 \\
\hline Convencional & 12 & 8 & 11 & 9 & 0 & 9 & 11 \\
\hline \multirow[t]{2}{*}{ Total } & 15 & 25 & 16 & 23 & 1 & 9 & 31 \\
\hline & \multicolumn{2}{|c|}{$\mathrm{CC}=0.421, \mathrm{p}<0.05$} & \multicolumn{3}{|c|}{$\mathrm{CC}=0.313, \mathrm{p}<0.05$} & \multicolumn{2}{|c|}{$\mathrm{CC}=0.474, \mathrm{p}<0.05$} \\
\hline
\end{tabular}

representar AO-OTA fracturas tipo 43 A2. Estos artículos revelaron que no había diferencia significativa en la rigidez, una tasa de unión de $92 \%$ con buenos a excelentes resultados funcionales en el tratamiento de fracturas de tibia distal con MIPO. ${ }^{7,19}$

Junto a los datos radiológicos para poder considerar una fractura curada hay que contar con el tiempo de evolución, el cual deberá ser adecuado para cada tipo de fractura. Asimismo, necesitamos la confirmación clínica como la ausencia de dolor y de movilidad del foco, con base en esto correlacionamos dos importantes estudios determinados de la escala de Montoya, que es útil para valorar el grado de consolidación, así como determinar que un grado 3 con ausencia de dolor y adecuada escala funcional es suficiente para comprobar que la fractura está consolidada..$^{20,21}$

Teniendo la corroboración clínico radiográfica de consolidación es importante establecer si dicho proceso generó alteración en las fases de la marcha, en el balance tanto sagital como coronal de la articulación de tobillo en relación a la pierna, determinar que los arcos de flexión y extensión son suficientes para evitar una artrosis postraumática inmediata así como de mediano y largo plazo. ${ }^{7,22}$ A partir de este momento la funcionalidad toma el papel protagónico, con esto nos referimos que un adecuado proceso quirúrgico que llegó a la consolidación debe evitar que ningún paciente presente dolor, una adecuada incorporación de la marcha que permita realizar actividades de la vida diaria, por ello sociedades internacionales de cirugía de pie y tobillo lograron un consenso con la escala funcional AOFAS que valora de 0 a 100 puntos, permitiéndonos inferir qué tratamiento se recomienda para una apropiada evolución natural de la enfermedad traumática de tibia distal, siendo adecuados los valores que se encuentren entre 80 y 100 puntos. ${ }^{10,23,24,25}$

Wenger R y colaboradores ${ }^{9}$ realizaron estudios con dos grupos donde compararon fracturas de tibia distal tratadas con técnica MIPO y fijación abierta convencional, todas ellas con placa anterolateral y en los cuales se obtuvieron datos relevantes a favor de la técnica MIPO con consolidación ósea y mayor puntaje funcional articular a los seis meses.

Estos resultados son similares a los de metaanálisis reportados en diversas sociedades europeas y estadounidenses de cirugía de pie y tobillo a partir de 2013.
Se encontró que la técnica MIPO no tuvo una superioridad estadísticamente significativa en la disminución de la frecuencia de infección postquirúrgica en las primeras seis semanas comparada con la técnica convencional abierta.

Al mismo tiempo observamos que la técnica MIPO reporta menor incidencia en dehiscencia de herida quirúrgica estadísticamente significativa en la semana dos postquirúrgica aportando de manera positiva para nuestro estudio.

Además de manera puntual se demostró que la técnica MIPO fue estadísticamente significativa sobre la técnica convencional para disminuir el tiempo de consolidación ósea radiográfica en los primeros seis meses postquirúrgicos.

Al mismo tiempo encontramos una fuerte correlación estadísticamente significativa con la técnica MIPO y puntajes de funcionalidad articular a partir de la semana seis con progresiva mejoría hasta el mes seis postquirúrgico, dato que es de gran relevancia en nuestro estudio.

Referencias

1. Kuo LT, Chi CC, Chuang CH. Surgical interventions for treating distal tibial metaphyseal fractures in adults. Cochrane Database Syst Rev. 2015; (3): CD010261.

2. Lister J. Antiseptic principle in the practice of surgery. $\mathrm{Br}$ Med J. 1867; 1: 1-3.

3. Bartoníbek J. Early history of operative treatment of fractures. Arch Orthop Trauma Surg. 2010; 130: 1385-96.

4. Hernigou P, Pariat J. History of internal fixation (part 1): early developments with wires and plates before World War II. Int Orthop. 2017; 41 (16): 1273-83.

5. Gautier E, Perren SM, Cordey J. Effect of plate position relative to bending direction on the rigidity of a plate osteosynthesis. A theoretical analysis. Injury. 2000; 31(Suppl. 3): 14-20.

6. Augata P, Von Rüden C. Evolution of fracture treatment with bone plates. Injury. 2018; 49(Suppl 1): S2-7.

. Lai TC, Fleming JJ. Minimally invasive plate osteosynthesis for distal tibia fractures. Clin Podiatr Med Surg. 2018; 35(2): 223-2.

8. Lakhotia D, Sharma G, Khatri K, Kumar GN, Sharma V, Farooque K. Minimally invasive osteosynthesis of distal tibial fractures using anterolateral locking plate: Evaluation of results and complications. Chin J Traumatol. 2016; 19(1): 39-44.

9. Wenger R, Oehme F, Winkler J, Perren SM, Babst R, Beeres FJP. Absolute or relative stability in minimal invasive plate osteosynthesis of simple distal meta or diaphyseal tibia fractures? Injury. 2017; 48(6): 1217-23.

10. Piatkowski K, Piekarczyk P, Kwiatkowski K, Przybycień M, Chwedczuk B. Comparison of different locking plate fixation methods in distal tibia fractures. Int Orthop. 2015; 39(11): 2245-51. 
11. Gülabi D, Bekler HI, Saglam F, Tasdemir Z, Cecen GS, Elmali N. Surgical treatment of distal tibia fractures: open versus MIPO. Ulus Travma Acil Cerrahi Derg. 2016; 22(1): 52-7.

12. Li Y, Jiang X, Guo Q, Zhu L, Ye T, Chen A. Treatment of distal tibial shaft fractures by three different surgical methods: a randomized, prospective study. Int Orthop. 2014; 38(6): 1261-7.

13. Zou J, Zhang W, Zhang CQ. Comparison of minimally invasive percutaneous plate osteosynthesis with open reduction and internal fixation for treatment of extra-articular distal tibia fractures. Injury. 2013; 44(8): 1102-6.

14. Viberg B, Kleven S, Hamborg-Petersen E, Skov O. Complications and functional outcome after fixation of distal tibia fractures with locking plate. Injury. 2016; 47(7): 1514-8.

15. Villamil GCE, González VJA, Velasco BA, Martínez AM, Fuentes R. Fracturas de extremo distal de tibia tratadas con placas de estabilidad angular. Estudio retrospectivo observacional entre técnica abierta y mínimamente invasiva. Revista del Pie y Tobillo. 2015; 29(1): 11-6.

16. Toogood P, Huang A, Siebuhr K, Miclau T. Minimally invasive plate osteosynthesis versus conventional open insertion techniques for osteosynthesis. Injury. 2018; 49(Suppl 1): S19-23.

17. Book: Danis R. Theorie et practique de l'osteosynthese. Paris: Masson; 1949.

18. Li A, Wei Z, Ding H, Tang H, Liu Y, Shi J, et al. Minimally invasive percutaneous plates versus conventional fixation techniques for distal tibial fractures: a meta-analysis. Int J Surg. 2017; 38: 52-60.
19. Jain D, Selhi HS, Yamin M, Mahindra P. Soft tissue complications in distal tibial fractures managed with medial locking plates: A myth or reality? J Clin Orthop Trauma. 2017; 8(Suppl 2): S90-5.

20. Calderón-Garcidueñas J, Castillo-Carranza MJ, Pavón-Salas D, Mireles-Díaz JA, González-Gutiérrez R. Fracturas con retardo en la consolidación ósea o pseudoartrosis: tratamiento no invasivo con electroestimulación galvánica transcutánea. Acta Ortop Mex. 2001; 15(6): 262-5.

21. Pirogova T, Bartolome VA, Bartolome VMJ. Valoración radiológica de consolidación de fracturas. Radiología. 2014; 56 (Espec Cong): 878.

22. Barcak E, Collinge CA. Metaphyseal distal tibia fractures: a cohort, single-surgeon study comparing outcomes of patients treated with minimally invasive plating versus intramedullary nailing. $J$ Orthop Trauma. 2016; 30(5): 169-74.

23. Fang JH, Wu YS, Guo XS, Sun LJ. Comparison of 3 minimally invasive methods for distal tibia fractures. Feature Article. 2016; 39: e627-33.

24. Madeley NJ, Wing KJ, Topliss C, Penner MJ, Glazebrook MA, Younger AS. Responsiveness and validity of the SF-36, Ankle Osteoarthritis Scale, AOFAS Ankle Hindfoot Score, and Foot Function Index in end stage ankle arthritis. Foot Ankle Int. 2012; 33(1): 57-63.

25. Agel J, Beskin JL, Brage M, Guyton GP, Kadel NJ, Saltzman CL, et al. Reliability of the foot function index: a report of the AOFAS outcomes committee. Foot Ankle Int. 2005; 26(11): 962-7.

Conflicto de intereses: Ninguno. 